\title{
DENSENESS OF OPERATORS WHICH ATTAIN THEIR NUMERICAL RADIUS
}

\author{
I. D. BERG' ${ }^{1}$ and BRAILEY SIMS
}

(Received 20 December 1982)

Communicated by R. O. Vyborny

\begin{abstract}
We show that a bounded linear operator on a uniformly convex space may be perturbed by a compact operator of arbitrarily small norm to yield an operator which attains its numerical radius.
\end{abstract}

1980 Mathematics subject classification (Amer. Math. Soc.): 47 A 12.

The second author, in his dissertation [Sims, 1972], raised the question of the norm-denseness of those operators which attained their numerical radii, paralleling the norm-attaining operator investigations of Lindenstrauss [1963] and the related results of Bishop and Phelps [1961]. He observed there that self-adjoint operators on a Hilbert space could be approximated by self-adjoint operators which attained their numerical radii.

In this note we show that any bounded operator on a Hilbert space can be approximated in norm by operators which attain their numerical radii and, incidentally, differ from the original operator by a compact operator. Indeed, our argument establishes this result for any uniformly convex Banach space.

It is not clear how far the argument or result extend. The numerical radius is a natural object of contemplation for Hilbert space but is less accessible for general Banach spaces. At present we do not know a counterexample to the theorem on any Banach space.

We acknowledge valuable correspondence with J. Williams who, in particular, pointed out directions of investigation which are not pursued in this paper but indicate promising lines for future investigation.

\footnotetext{
'The first author's research was partially supported by the National Science Foundation.

(C) 1984 Australian Mathematical Society 0263-6115/84 \$A2.00+0.00
} 
We let $X$ be a Banach space, $X^{*}$ its dual space and $B(X)$ the algebra of bounded linear operators on $X$.

Let

$$
\Pi:=\left\{(x, f) \in X \times X^{*}: f(x)=\|f\|=\|x\|=1\right\} .
$$

Recall: The numerical radius of $T \in B(X)$ is

$$
\nu(T):=\operatorname{Sup}\{|f(T x)|:(x, f) \in \Pi\}
$$

and we say $T$ attains its numerical radius if there exists $\left(x_{0}, f_{0}\right) \in \Pi$ with

$$
\left|f_{0}\left(T x_{0}\right)\right|=\nu(T) \text {. }
$$

(Technical) Lemma. For $X$ a normed linear space and $T \in B(X)$, given constants $a_{1}, b, a_{2}>0$ and $\left(x_{1}, f_{1}\right) \in \Pi$ with $\left|f_{1}\left(T x_{1}\right)\right|>\nu(T)-a_{1}$, there exists $T^{\prime} \in B(X)$ with

$$
T^{\prime}-T \text { a rank-one operator of norm } b,
$$

and there exists $\left(x_{2}, f_{2}\right) \in \Pi$ with

$$
\left|f_{2}\left(T^{\prime} x_{2}\right)\right|>\nu\left(T^{\prime}\right)-a_{2}
$$

and

$$
f_{1}\left(x_{2}\right)>1-\frac{a_{1}+a_{2}}{b} \text {. }
$$

Moreover,

$$
\left\|\frac{x_{1}+x_{2}}{2}\right\|>1-\frac{a_{1}+a_{2}}{2 b} .
$$

Proof. (1) is satisfied by taking

$$
T^{\prime}(x)=T(x)+b e^{i \theta} f_{1}(x) x_{1}
$$

for all $x \in X$, where $\theta=\arg f_{1}\left(T x_{1}\right)$.

Now choose $\left(x_{2}, f_{2}\right) \in \Pi$ such that (2) is satisfied and simultaneously $f_{1}\left(x_{2}\right) \geqslant$ 0 . This is possible since $(x, f) \in \Pi$ implies $(\lambda x, \bar{\lambda} f) \in \Pi$ for any $\lambda$ with $|\lambda|=1$. To establish (3) note that

$$
\begin{aligned}
\nu\left(T^{\prime}\right)-a_{2} & <\left|f_{2}\left(T^{\prime} x_{2}\right)\right|=\left|f_{2}\left(T x_{2}\right)+b e^{i \theta} f_{1}\left(x_{2}\right) f_{2}\left(x_{1}\right)\right| \\
& \leqslant\left|f_{2}\left(T x_{2}\right)\right|+b\left|f_{1}\left(x_{2}\right)\right|\left|f_{2}\left(x_{1}\right)\right| \\
& \leqslant \nu(T)+b f_{1}\left(x_{2}\right) \quad \text { as }\left|f_{1}\left(x_{2}\right)\right|=f_{1}\left(x_{2}\right) \text { and }\left|f_{2}\left(x_{1}\right)\right| \leqslant 1 .
\end{aligned}
$$

Further,

$$
\begin{aligned}
\nu\left(T^{\prime}\right) & \geqslant\left|f_{1}\left(T^{\prime} x_{1}\right)\right|=\left|f_{1}\left(T x_{1}\right)+b e^{i \theta}\right| \\
& =\left|f_{1}\left(T x_{1}\right)\right|+b \quad(\text { by choice of } \theta) \\
& \geqslant \nu(T)-a_{1}+b .
\end{aligned}
$$


Combining these yields

$$
\nu(T)-a_{1}+b-a_{2}<\nu(T)+b f_{1}\left(x_{2}\right)
$$

or

$$
f_{1}\left(x_{2}\right)>1-\frac{a_{1}+a_{2}}{b} \text { as required. }
$$

We now establish (4) by noting

$$
\left\|\frac{x_{1}+x_{2}}{2}\right\| \geqslant f_{1}\left(\frac{x_{1}+x_{2}}{2}\right) \geqslant \frac{1}{2}\left(1+1-\frac{a_{1}+a_{2}}{b}\right)=1-\frac{a_{1}+a_{2}}{2 b} .
$$

Now let $X$ be uniformly convex with modulus of convexity

$$
\delta(\varepsilon):=\inf \left\{1-\left\|\frac{x+y}{2}\right\|:\|x\|=\|y\|=1 \text { and }\|x-y\| \geqslant \varepsilon\right\} .
$$

Then $\delta(\varepsilon)>0$ for all $\varepsilon>0$ and $\|x-y\|<\varepsilon$ whenever $\|(x+y) / 2\|>1-\delta(\varepsilon)$. Let $\varepsilon>0$ be given. Choose positive sequences $\left(\varepsilon_{n}\right)$ and $\left(\varepsilon_{n}^{\prime}\right)$ such that: $\varepsilon_{n}$ is monotonically decreasing to $0 ; \sum \varepsilon_{n}^{\prime}<\varepsilon$ and

$$
\frac{\varepsilon_{n}}{\varepsilon_{n}^{\prime}}<\delta\left(\frac{1}{2^{n}}\right)
$$

[For example, take $\varepsilon_{n}^{\prime}=\varepsilon / 2^{n}$ and $\varepsilon_{n}=\left(\varepsilon \delta\left(1 / 2^{n}\right)\right) / 2^{n+1}$.]

Using the above lemma we may inductively construct sequences $\left(T_{n}\right)$ and $\left(x_{n}, f_{n}\right) \in \Pi$ as follows: $T_{1}=T$ and $\left(x_{1}, f_{1}\right)$ is such that $\left|f_{1}\left(T_{1} x_{1}\right)\right|>\nu\left(T_{1}\right)-\varepsilon_{1}$; $T_{2}$ is such that $T_{2}-T_{1}$ is a rank-one operator of norm $\varepsilon_{1}^{\prime}$ and $\left(x_{2}, f_{2}\right)$ is such that $\left|f_{2}\left(T_{2} x_{2}\right)\right|>\nu\left(T_{2}\right)-\varepsilon_{2}$ and

$$
\left\|\frac{x_{1}+x_{2}}{2}\right\|>1-\frac{\varepsilon_{1}+\varepsilon_{2}}{2 \varepsilon_{1}^{\prime}}>1-\frac{\varepsilon_{2}}{\varepsilon_{1}^{\prime}}>1-\delta\left(\frac{1}{2}\right) .
$$

So $\left\|x_{2}-x_{1}\right\|<\frac{1}{2}$ [take $a_{1}=\varepsilon_{1}, b=\varepsilon_{1}^{\prime}$ and $a_{2}=\varepsilon_{2}$ ]; and in general: $T_{n}$ is such that $T_{n}-T_{n-1}$ is a rank-one operator of norm $\varepsilon_{n}^{\prime}$ and $\left(x_{n}, f_{n}\right)$ is such that $\left|f_{n}\left(T_{n} x_{n}\right)\right|>\nu\left(T_{n}\right)-\varepsilon_{n}$ and $\left\|x_{n}-x_{n-1}\right\|<1 / 2^{n}$. It now follows that $\left(T_{n}\right)$ is a Cauchy sequence $\left[\left\|T_{m}-T_{n}\right\| \leqslant \sum_{k=n+1}^{m}\left\|T_{k}-T_{k-1}\right\| \leqslant \sum_{k=n+1}^{m} \varepsilon_{k}^{\prime} \rightarrow 0\right.$ as $m>n \rightarrow$ $\infty$ ] as is $\left(x_{n}\right)$. Thus $T_{n}$ converges to $T_{\infty} \in B(X)$ and $x_{n} \rightarrow x_{\infty}$ where $\left\|x_{\infty}\right\|=1$ and $T_{\infty}-T$ is a compact operator of norm less than or equal to $\sum \varepsilon_{n}^{\prime}<\varepsilon$. By passing to a subsequence if necessary we may assume that $f_{n}$ converges weakly to $f_{\infty}$ with $\left\|f_{\infty}\right\| \leqslant 1$. ( $X$ is reflexive, so the unit ball of $X^{*}$ is weakly compact.)

We show $\left(x_{\infty}, f_{\infty}\right) \in \Pi$ and $\left|f_{\infty}\left(T_{\infty} x_{\infty}\right)\right|=\nu\left(T_{\infty}\right)$. Now,

$$
\begin{aligned}
f_{\infty}\left(x_{\infty}\right) & =\lim f_{n}\left(x_{\infty}\right)=\lim \left(f_{n}\left(x_{n}\right)+f_{n}\left(x_{\infty}-x_{n}\right)\right) \\
& =1, \quad \text { as } f_{n}\left(x_{n}\right)=1 \text { and }\left\|x_{\infty}-x_{n}\right\| \rightarrow 0 .
\end{aligned}
$$


So $\left(x_{\infty}, f_{\infty}\right) \in \Pi$. Further,

$$
\begin{aligned}
\nu\left(T_{\infty}\right) & =\lim \nu\left(T_{n}\right) & & \text { as }\left\|T_{n}-T_{\infty}\right\| \rightarrow 0 \\
& =\lim \left|f_{n}\left(T_{n} x_{n}\right)\right| & & \text { as } \nu\left(T_{n}\right)-\left|f_{n}\left(T_{n} x_{n}\right)\right| \rightarrow 0 \\
& =\lim \left|f_{n}\left(T_{\infty} x_{\infty}\right)\right| & & \text { as }\left\|T_{n} x_{n}-T_{\infty} x_{\infty}\right\| \rightarrow 0 \\
& =\left|f_{\infty}\left(T_{\infty} x_{\infty}\right)\right| & & \text { as } f_{n} \stackrel{w}{\rightarrow} f_{\infty},
\end{aligned}
$$

and we have established:

THEOREM. For $X$ a uniformly convex Banach space, given $T \in B(X)$ and $\varepsilon>0$, there exists a compact operator $C$ with $\|C\|<\varepsilon$ and $(x, f) \in \Pi$ such that $\nu(T+C)$ $=|f((T+C) x)|$.

That is; $T$ may be perturbed by a compact operator of arbitrarily small norm to obtain an operator which attains its numerical radius. In particular, the operators which attain their numerical radius are norm dense in $B(X)$. We remark that, as a consequence of a theorem of Weyl [Halmos, 1967, problem 43], in the case of a self-adjoint operator on a Hilbert space the necessary perturbation can be realized by a rank-one self-adjoint operator.

\section{References}

Errett Bishop and R. R. Phelps, 'A proof that every Banach space is subreflexive', Bull. Amer. Math. Soc. 67 (1961), 97-98.

P. Halmos, A Hilbert space problem book (Van Nostrand, New York, 1967).

Joram Lindenstrauss, 'On operators which attain their norm', Israel J. Math. 1 (1963), 139-148.

Brailey Sims, On numerical range and its application to Banach algebra (Ph.D. dissertation, University of Newcastle, Australia, 1972).

Department of Mathematics

University of Illinois at Urbana-Champaign

273 Altgeld Hall, 1409 West Green Street

Urbana, Illinois 61801

U.S.A.
Department of Mathematics

University of New England

Armidale, N.S.W. 2351

Australia 